\title{
Bemerkung zu Jacobis Beweis für die Anzahl der Doppeltangenten.
}

(Von Herrn A. Clebsch zu Giessen.)

Der schöne Beweis, welchen Jacobi für die Anzahl der Doppeltangenten einer algebraischen Curve geliefert hat, wird, wie ich glaube, im Allgemeinen nicht in demjenigen Grade gewürdigt und gekannt, als er es verdient; und es liegt dies vielleicht an der Darstellung, welche in einzelnen Punkten nicht so symmetrisch ist, als man es heute bei durchgängiger Anwendung homogener Coordinaten zu verlangen pflegt, und welche namentlich an einer Stelle den einfachen Grundgedanken ein wenig verdeckt. Ich habe versucht, für meine Vorlesungen an der hiesigen Universität, diesen Beweis etwas zu modificiren, und ich glaube, dass dadurch der Grundgedanke desselben klar und einfach hervortritt.

Sei $x$ der Berührungspunkt einer Doppeltangente der Curve $n^{\text {ter }}$ Ordnung $u=0$, und $y$ ein Punkt, welcher gleichzeitig auf der Tangente von $x$ :

(1.) $u_{1} y_{1}+u_{2} y_{2}+u_{3} y_{3}=0$,

$\left(u_{i}=\frac{1}{n} \frac{\partial u}{\partial x_{i}}\right)$, und auf einer beliebig gewählten Geraden

(2.) $\quad c_{1} y_{1}+c_{2} y_{2}+c_{3} y_{3}=0$

sich befindet. Stellt man dann $u$ symbolisch durch

$$
\left(a_{1} x_{1}+a_{2} x_{2}+a_{3} x_{3}\right)^{n}
$$

dar, so ist die Bedingung dafür, dass $x$ Berührungspunkt einer Doppeltangente werde, diese, dass die aus der Gleichung

$$
\text { (3.) } \quad\left(\mu\left(a_{1} x_{1}+a_{2} x_{2}+a_{3} x_{3}\right)+\lambda\left(a_{1} y_{1}+a_{2} y_{2}+a_{3} y_{3}\right)\right)^{n}=0,
$$

nach Absonderung des Factors $\lambda^{2}$ gebildete Discriminante verschwinde. 
Eliminirt man nun die $y$ mit Hülfe von (1.), (2.), so erhält man aus (3.):

$$
\text { (4.) } \quad\left(\mu\left(a_{1} x_{1}+a_{2} x_{2}+a_{3} x_{3}\right)+\lambda\left|\begin{array}{lll}
a_{1} & u_{1} & c_{1} \\
a_{2} & u_{2} & c_{2} \\
a_{3} & u_{3} & c_{3}
\end{array}\right|\right)^{n}=0 \text {. }
$$

Die fragliche Discriminante sei $F(x, c)=0$; es ist zu beweisen, dass aus dieser Gleichung sich der Factor $c_{1} x_{1}+c_{2} x_{2}+c_{3} x_{3}$ so oft muss absondern lassen, dass die $c$ im Rest nicht mehr vorkommen. Dass der übrigbleibende Factor dann von der Ordnung $(n-2)\left(n^{2}-9\right)$ ist, zeigt eine Abzählung.

Setzen wir nun

$$
\lambda=\varrho\left(b_{1} x_{1}+b_{2} x_{2}+b_{3} x_{3}\right)
$$

wo die $b$ wieder ganz beliebige Grössen sind; und bezeichnen wir der Kürze wegen Ausdrücke wie $b_{1} x_{1}+b_{2} x_{2}+b_{3} x_{3}$ durch $b$. Dann ist identisch

$$
0=\left|\begin{array}{llll}
a_{1} & b_{1} & c_{1} & u_{1} \\
a_{2} & b_{2} & c_{2} & u_{2} \\
a_{3} & b_{3} & c_{3} & u_{3} \\
a & b & c & u
\end{array}\right|=a A-b B+c C
$$

Die Gleichung (4.), welche man durch.

$$
(\mu a+\lambda B)^{n}=0
$$

ersetzen kann, geht so über in

$$
(\mu a+\rho b B)^{n}=0,
$$

oder mit Hülfe der soeben entwickelten Gleichung in

$$
((\mu+\varrho A) a+\varrho c C)^{n}=0 .
$$

Führt man also statt $\lambda, \mu$ die Grössen

$$
\begin{aligned}
\mu^{\prime} & =\mu+\varrho A=\mu+\frac{\lambda A}{b}, \\
\lambda^{\prime} & =\rho c=\frac{\lambda c}{b}
\end{aligned}
$$

ein, so erhält man die Gleichung

$$
\left(\mu^{\prime} a+\lambda^{\prime} C\right)^{n}=0
$$


welche sich von der ursprünglichen nur dadurch unterscheidet, dass die $b$ an Stelle der $c$ getreten sind. Diese Gleichung, durch $\lambda^{\prime 2}$ dividirt, giebt also die Discriminante $F(x, b)=0$. Und da beide Discriminanten sich nur durch eine Potenz der Transformationsdeterminante unterscheiden können, die hier $\frac{c}{b}$ ist, so hat man

$$
F(x, c)=\left(\frac{c}{b}\right)^{\alpha} \cdot F(x, b), \quad \frac{F(x, c)}{c^{\alpha}}=\frac{F(x, b)}{b^{\alpha}},
$$

diese Quotienten müssen also von den $c$ frei werden, was zu beweisen war.

Giessen, den 23. Juli 1863. 


\section{Crelle Journal f Math. 63 r Band 2. Heft.}
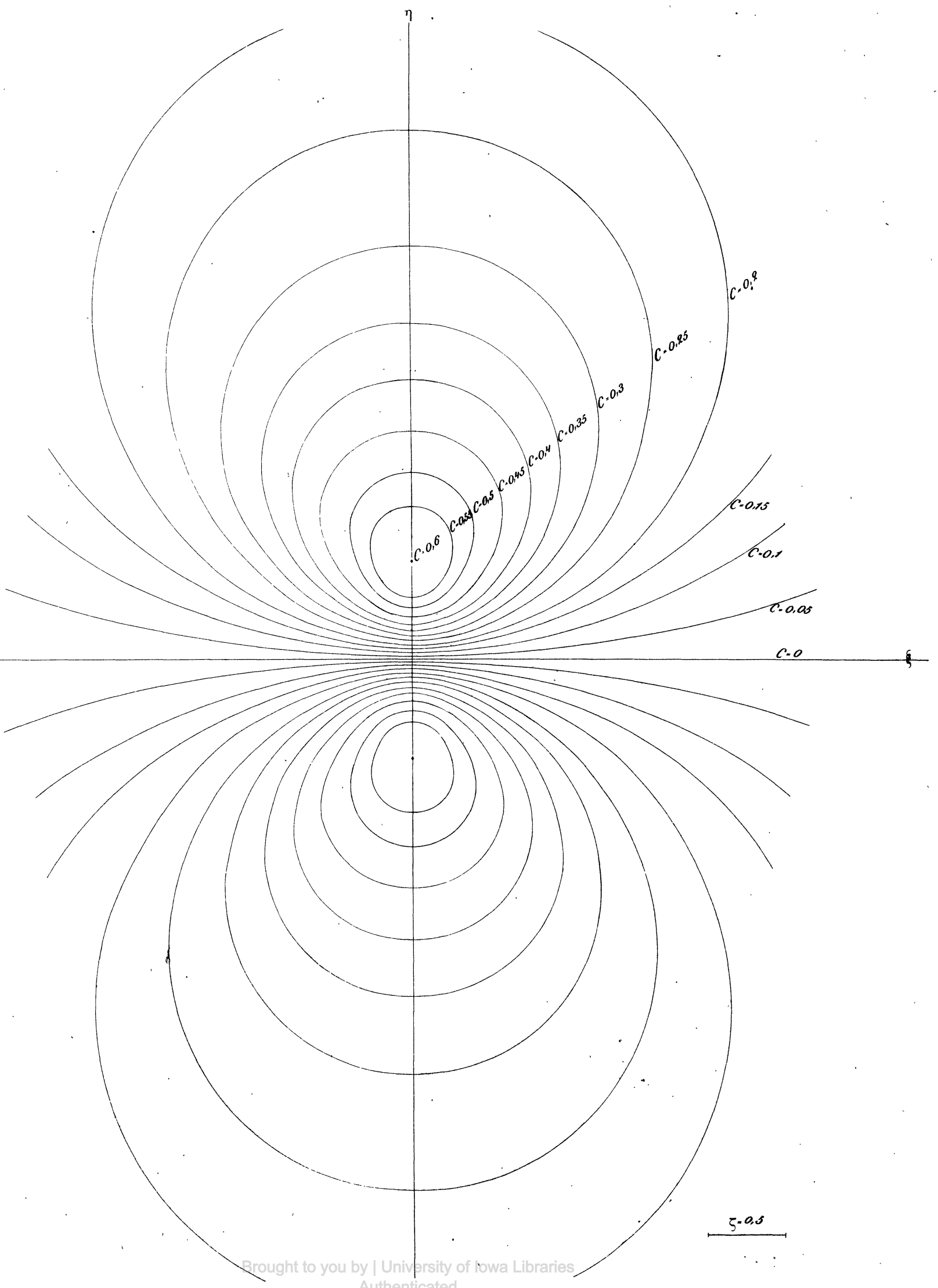
1 